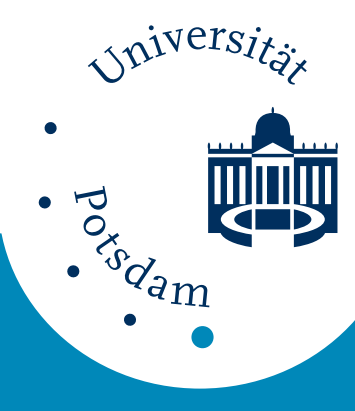

Universität Potsdam

Carsten Allefeld, Jürgen Kurths

\title{
An Approach to Multivariate Phase Synchronization Analysis and its Application to Event-Related Potentials
}

first published in:

International Journal of Bifurcation and Chaos. - 14 (2004), 2, pp. 417 - 426

ISSN (print) 0218-1274

ISSN (online) 1793-6551

DOI 10.1142/S0218127404009521

Postprint published at the Institutional Repository of the

Potsdam University:

In: Postprints der Universität Potsdam :

Mathematisch-Naturwissenschaftliche Reihe ; 69

http://opus.kobv.de/ubp/volltexte/2008/2010/

http://nbn-resolving.de/urn:nbn:de:kobv:517-opus-20106

Postprints der Universität Potsdam

Mathematisch-Naturwissenschaftliche Reihe ; 69

ISSN 1866-8372 


\title{
An Approach to Multivariate Phase Synchronization Analysis and its Application to Event-Related Potentials
}

- Synchronization Cluster Analysis -

\author{
Carsten Allefeld Jürgen Kurths
}

Nonlinear Dynamics Group, Institute of Physics, and Research Group “Conflicting Rules", Universität Potsdam, Am Neuen Palais 19, PF 601553, D-14415 Potsdam, Germany

allefeld@ling.uni-potsdam.de

Electronic version of an article published as: International Journal of Bifurcation and Chaos, 14(2), 2004, 417-426. doi:10.1142/S0218127404009521 (C) World Scientific Publishing Company http:/ / www.worldscinet.com/ijbc/ijbc.shtml

\begin{abstract}
A method for the multivariate analysis of statistical phase synchronization phenomena in empirical data is presented. A first statistical approach is complemented by a stochastic dynamic model, to result in a data analysis algorithm which can in a specific sense be shown to be a generic multivariate statistical phase synchronization analysis. The method is applied to EEG data from a psychological experiment, obtaining results which indicate the relevance of this method in the context of cognitive science as well as in other fields.
\end{abstract}




\section{Introduction}

Synchronization phenomena are a classic subject of science (cf. Pikovsky et al. [2001]). In the last years especially the investigation of phase synchronization has received much attention following the study of Rosenblum et al. [1996], who showed that there is a specific regime in the dynamics of two coupled chaotic oscillators in which the phase difference of the oscillators is bounded while the amplitudes remain uncorrelated and irregular. Phase synchronization effects have been studied numerically, in laboratory experiments [Parlitz et al., 1996; Ticos et al., 2000; Kiss \& Hudson, 2001] as well as in data obtained from natural systems including the human brain [Mormann et al., 2000; Lachaux et al., 1999]. In cases like this, where the system dynamics is very complex and the data are noisy, the definition of phase synchronization has to be given a quantitative statistical form, measuring the "peakedness" of the distribution of the generalized phase difference $\Delta \varphi=m \varphi_{2}-n \varphi_{1}$ of two oscillators [Tass et al., 1998].

Until now, such statistical phase synchronization analysis has been constrained to the bivariate case, while the examination of empirical multivariate data was accomplished by the simple repeated application of bivariate synchronization measures. For instance, Rodriguez et al. [1999] tested for significant increases and decreases in the strength of phase synchronization between signals obtained from scalp EEG recordings in a visual attention task, separately for each pair of electrodes, and displayed the results as colored lines between the sites in a schematic map of the scalp. This approach gives detailed information on the topographic structure of synchronization relations, but it has at least two drawbacks: The visualization can get incomprehensible if a large number of lines has to be drawn, and this analysis in itself gives no information on a common integrating structure that may be present in the data. In the other extreme, Haig et al. [2000] computed an index of global phase synchronization which is meant to indicate synchronization phenomena between all recording sites at once, but fails to give topographic details and effectively destroys much of the information present in the data.

In this paper, we present an approach to genuinely multivariate phase synchronization analysis that tries to combine the global with the topographically detailed perspective. To this end, we introduce the concept of a statistical phase synchronization cluster and derive a method to identify this structure in a given data set. In contrast to other studies concerning the dynamics and stability of clusters of perfect (phase) synchronization and the coexistence and interaction of multiple clusters (cf. e.g. Osipov \& Kurths [2001]; Osipov et al. [1997]), the present paper tries to describe the form of a single statistical cluster, in which the oscillators participate in different degrees, ranging from no to perfect agreement with the cluster dynamics. Its goal is to derive a general structure whose application to empirical data can be seen as a generic multivariate analysis in the field of phase synchronization.

In the following mathematical derivations we will frequently refer to concepts coming from the context of directional statistics. A short overview of this is given by Allefeld \& Kurths [this issue]; for a comprehensive introduction, see the monographs by Mardia [Mardia \& Jupp, 2000; Mardia, 1972].

\section{Synchronization Cluster Analysis}

In statistical phase synchronization analysis, the relevant information is given by the phase $\varphi_{i k}$ of the $N$ oscillators $i=1 \ldots N$ in a number of realizations $k=1 \ldots n$ of the stochastic process considered. The strength of synchronization between each two oscillators can be measured by a statistic on the distribution of their phase 
difference; here we use the quantity

$$
\bar{R}_{i j}=\left|\frac{1}{n} \sum_{k} \exp \left(\mathrm{i}\left(\varphi_{j k}-\varphi_{i k}\right)\right)\right| .
$$

It takes on values in the range from 0 (no phase synchronization) to 1 (perfect phase synchronization). The objective of multivariate phase synchronization analysis is to derive from this matrix $\left(\bar{R}_{i j}\right)$ of bivariate indices some information about the synchronization state of the whole of $N$ oscillators.

In the following sections, we will present our approach, the synchronization cluster analysis, in three steps. The general definition of a synchronization cluster is followed by its concretization in a very specific dynamical model, which motivates a generally applicable method of data analysis. Each step does not directly derive from the following, but introduces a significant modification.

\subsection{The concept of a synchronization cluster}

The basic idea of multivariate synchronization analysis introduced in this paper is to conceive of the oscillators as constituting a cluster in which they participate in different degrees $c_{i}$. The cluster consists of a common rhythm, a mean of the oscillations of the single oscillators, and it is described by the dynamics of a cluster phase $\Phi$.

In each realization, this reference phase of the cluster is defined as a circular weighted mean of the oscillator phases,

$$
\Phi_{k}=\arg \sum_{j} c_{j} \exp \left(\mathrm{i} \varphi_{j k}\right),
$$

while the participation indices $c_{i}$ are calculated as a (monotonously increasing) function of the synchronization strength between an oscillator and the cluster,

$$
c_{i}=f\left(\bar{R}_{i \mathrm{C}}\right) \quad \text { with } \quad \bar{R}_{i \mathrm{C}}=\left|\frac{1}{n} \sum_{k} \exp \left(\mathrm{i}\left(\varphi_{i k}-\Phi_{k}\right)\right)\right| .
$$

In this way, the participation index quantifies both how close an oscillator follows the common rhythm as well as how important it is in its contribution to the cluster.

A self-consistent solution of this set of equations would represent a synchronization cluster analysis of the given data set $\varphi_{i k}$. A problem for this ansatz is that while the definition of the cluster phase is quite straightforward, it is not immediately obvious which function $f$ should be chosen for the relation between the $\bar{R}_{i C}$ and the $c_{i}$.

\subsection{A dynamical perspective}

To fill this gap, a look at the process leading to the formation of a synchronization cluster may be helpful. The model we are looking at does not maintain the generality of the first approach, but clarifies the dynamical meaning of the statistical quantities introduced above. As we will see, this concretization will also lead to a modification of the given ansatz.

We use the following model of $N$ coupled noisy phase oscillators as a starting point:

$$
\dot{\varphi}_{i}=\omega_{i}+\sum_{j} k_{i j} \sin \left(\varphi_{j}-\varphi_{i}\right)+\xi_{i} .
$$

It derives from the well-known Kuramoto model [Strogatz, 2000] with two important differences: The strength of the coupling between two oscillators $k_{i j}$ is not the 
same for each pair, and the dynamics has been complemented by a stochastic part. The $\xi_{i}$ are taken to be independent Gaussian white noises with the same energy, normalized to 1 by an appropriate choice of the time unit.

In the case that the coupling matrix factorizes, $k_{i j}=c_{i} c_{j}$, the differential equations can be decoupled by a mean field approach such that

$$
\dot{\varphi}_{i}=\omega_{i}+c_{i} M \sin \left(\Phi-\varphi_{i}\right)+\xi_{i},
$$

where

$$
M=\left|\sum_{j} c_{j} \exp \left(\mathrm{i} \varphi_{j}\right)\right| \quad \text { and } \Phi=\arg \sum_{j} c_{j} \exp \left(\mathrm{i} \varphi_{j}\right)
$$

are the amplitude and the phase of the mean field, respectively. By this transformation, the coupling between oscillators has been formally replaced by a coupling to the mean field. If the number of oscillators is large enough and the system is in its stationary state, the mean field is approximately independent of the dynamics of the individual oscillators, and so it can be treated as a common external driving with a constant amplitude $M=\langle M\rangle$.

If the variation of the autonomous frequencies $\omega_{i}$ is small, the difference between them and the frequency of the mean field $\dot{\Phi}$ can be neglected, and the dynamics of the phase difference between an oscillator and the mean field, $\Delta \varphi_{i}=$ $\varphi_{i}-\Phi$, obtains the form

$$
\Delta \dot{\varphi}_{i}=-c_{i} M \sin \left(\Delta \varphi_{i}\right)+\xi_{i}
$$

The stationary distribution resulting from this stochastic dynamics is a von Mises distribution, $\Delta \varphi_{i} \sim \mathrm{M}\left(0,2 c_{i} M\right)$, and the corresponding population value of the oscillator-mean field synchronization strength is

$$
\rho_{i \mathrm{C}}=\left|\left\langle\exp \left(\mathrm{i} \Delta \varphi_{i}\right)\right\rangle\right|=\mathrm{A}\left(2 c_{i} M\right) \quad \text { with } \quad \mathrm{A}(\kappa)=\frac{\mathrm{I}_{1}(\kappa)}{\mathrm{I}_{0}(\kappa)}
$$

where $\mathrm{I}_{p}$ denotes the modified Bessel function of the first kind of order $p$.

The parts of this dynamical model can be easily identified with those of the original approach. The synchronization cluster corresponds to the mean field, which effectively rules the dynamics of the individual oscillators and the cluster phase $\Phi_{k}$ is a realization of the phase of the mean field $\Phi$. The "participation" of an oscillator in the cluster is given by the coefficient $c_{i}$, which specifies its contribution as well as its coupling to the mean field. And the oscillator-cluster synchronization strength $\bar{R}_{i \mathrm{C}}$ is an empirical estimator of $\rho_{i \mathrm{C}}$, which depends on the participation index.

As a modification of the original model, the relation between $c_{i}$ and $\rho_{i \mathrm{C}}$ is not a simple function, but additionally depends on the amplitude of the mean field $M$, whose value in the stationary state is a result of the couplings of all oscillators in the cluster to each other. Because of the form of $M$ as a weighted sum it can be used to define a normalized index of the overall cluster strength

$$
r_{\text {Cluster }}=\frac{1}{\sum\left|c_{j}\right|} M=\left|\frac{1}{\sum\left|c_{j}\right|} \sum_{j} c_{j} \exp \left(\mathrm{i} \varphi_{j}\right)\right|
$$

(values from 0 to 1), which can be seen as a generalization of the global synchronization index $r_{\text {Global }}=\left|\frac{1}{N} \sum \exp \left(\mathrm{i} \varphi_{j}\right)\right|$ used by Haig et al. [2000]. Its expectation value can be expressed as a weighted mean of the $\rho_{i C}$,

$$
\left\langle r_{\text {Cluster }}\right\rangle=\frac{1}{\sum\left|c_{j}\right|} \sum_{j}\left|c_{j}\right| \rho_{j \mathrm{C}} .
$$




\subsection{The data analysis algorithm}

The dynamic approach did not lead to a simple specification of the function $f$, and the first definition of the cluster phase (Eq. 2) is only in a specific model identical to the phase of the mean field (Eq. 6). But the dynamical perspective enables us to modify the idea of synchronization cluster analysis in a way that is much more generally applicable (see below). It is based on another theoretical observation. For the population values of the bivariate synchronization indices $\bar{R}_{i j}$ holds

$$
\rho_{i j}=\left|\left\langle\exp \left(\mathrm{i}\left(\varphi_{j}-\varphi_{i}\right)\right)\right\rangle\right|=\left|\left\langle\exp \left(\mathrm{i}\left(\Delta \varphi_{j}-\Delta \varphi_{i}\right)\right)\right\rangle\right| \text {. }
$$

If in the given dynamics it is possible to introduce in some specific way a mean field, then the dynamics of the phase differences are decoupled. If additionally each oscillator is driven by noise independent of that acting on the other oscillators, then $\Delta \varphi_{i}$ and $\Delta \varphi_{j}$ become independent random variables, and so

$$
\rho_{i j}=\left|\left\langle\exp \left(\mathrm{i} \Delta \varphi_{j}\right)\right\rangle\right|\left|\left\langle\exp \left(-\mathrm{i} \Delta \varphi_{i}\right)\right\rangle\right|=\rho_{i \mathrm{C}} \rho_{j \mathrm{C}} \quad \text { for } \quad i \neq j \quad\left(\rho_{i i}=1\right),
$$

that is, the synchronization matrix (excepting the diagonal) factorizes.

This leads to a version of synchronization cluster analysis in which the quantity to be estimated from the data is no longer the participation index $c_{i}$, which depends on the specific dynamics, but the strength of the synchronization between an oscillator and the cluster $\rho_{i \mathrm{C}}$. Like $c_{i}$, this quantity is a measure of the degree of participation of the oscillator in the cluster. The corresponding algorithm is as follows: ${ }^{1} \bar{R}_{i j}$ is an empirical estimate of $\rho_{i j}=\rho_{i \mathrm{C}} \rho_{j \mathrm{C}}$ which can be shown to be asymptotically normally distributed, $\bar{R}_{i j} \sim \mathrm{N}\left(\rho_{i j}, \sigma_{i j}^{2}\right)$. A maximum likelihood estimation of the $\rho_{i \mathrm{C}}$ then reduces to minimizing the sum of square weighted errors

$$
\sum_{i, j>i} E_{i j}^{2} \text { with } E_{i j}=\frac{\bar{R}_{i j}-\rho_{i \mathrm{C}} \rho_{j \mathrm{C}}}{\sigma_{i j}},
$$

where

$$
\sigma_{i j}=\frac{1}{\sqrt{2 n}}\left(1-\rho_{i \mathrm{C}}^{2} \rho_{j \mathrm{C}}^{2}\right)
$$

is based on the assumption that due to the central limit theorem the difference of two independent circular random variables can in sufficiently good approximation be described by a wrapped normal distribution. The residual errors can then be used to check whether the model may be applied to the given data set.

In this form, synchronization cluster analysis is independent of most of the details of the dynamical model used for its motivation. The basic premises that are relevant to this approach are that the dynamics of the oscillators can be decoupled by introducing a mean field and that its stochastic part is independent for each of them. In this sense, the factorization of the matrix of bivariate synchronization indices $\bar{R}_{i j}$ by estimating the synchronization strengths to the cluster $\rho_{i \mathrm{C}}$ can be regarded as the generic multivariate phase synchronization analysis aimed at in the beginning. This genericity does not mean that it is necessarily applicable to every data set, since there still are specific assumptions-the feasibility of decoupling being the most important, because it underlies the unity of the synchronization cluster. But even in cases where the applied structure is not perfectly adequate, it may serve as a first approximation and specific deviations from the applied model may be detected by large values of the residual errors $E_{i j}$. Another favourable characteristic of this analysis is that its result maintains a direct relation

\footnotetext{
${ }^{1}$ This derivation is based on results of directional statistics, cf. Allefeld \& Kurths [this issue] or Mardia \& Jupp [2000].
} 


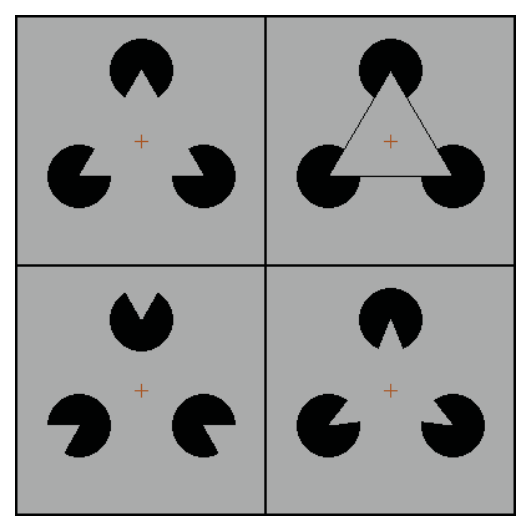

Figure 1: The stimuli: Kanizsa, Triangle, Non-Triangle, and Target. The cross at the center is used to suppress eye movements. The first three stimuli correspond to experimental conditions.

to the bivariate synchronization indices; an approximation of the synchronization strength between two oscillators $\bar{R}_{i j}$ is given by the product of the estimates of the synchronization strengths to the cluster $\rho_{i C} \rho_{j \mathrm{C}}$. In the following we will see that it can be successfully applied to an empirical data set.

\section{Application to EEG Data}

The analysis has been applied to event-related potentials recorded in a psychological experiment following Tallon-Baudry et al. [1996]. Data was obtained from one female subject of 23 years, right-handed and with normal vision. Four types of stimuli (Fig. 1) were presented in a randomized order for $700 \mathrm{~ms}$ with a random interstimulus interval of 2-3 seconds. The stimuli corresponding to experimental conditions consisted of a Kanizsa triangle with an illusory contour ("Kanizsa"), a similar shape with triangle edges drawn ("Triangle"), and a shape consisting of the same parts as the Kanizsa without forming an illusory contour ("Non-Triangle"). The task of the subject was to count silently the number of occurences of the fourth stimulus ("Target"), a variant of the Kanizsa, per experimental block. There were eight blocks of 90 stimulus presentations each.

EEG was recorded with a sampling rate of $500 \mathrm{~Hz}$ at 30 electrodes (see Fig. 5) and artifact-free epochs from $-300 \mathrm{~ms}$ to $650 \mathrm{~ms}$ relative to the stimulus presentation were selected for processing. To decrease the correlation between nearby electrodes and to obtain reference-free data, the scalp current density estimation procedure described by Perrin et al. [1989] was applied assuming idealized 10-20 positions on the unit sphere and with spline order $m=4$. The recorded time series $x(t)$ for each condition, electrode, and trial were convolved with a Morlet wavelet

$$
\psi\left(t ; \omega_{0}, \sigma_{\omega}\right)=\sqrt[4]{\frac{2}{\pi}} \sqrt{\sigma_{\omega}} \exp \left(-\sigma_{\omega}^{2} t^{2}\right) \exp \left(\mathrm{i} \omega_{0} t\right)
$$

to obtain complex wavelet coefficients

$$
w(t, f)=\int_{-\infty}^{\infty} x\left(t^{\prime}\right) \psi\left(t-t^{\prime} ; 2 \pi f, \frac{2 \pi f}{10}\right) \mathrm{d} t^{\prime}
$$

that were used to define frequency-specific instantaneous phases

$$
\varphi(t, f)=\arg w(t, f) .
$$


The convolution was performed via FFT assuming periodic boundary conditions, and the resulting data were reduced to epochs from $-150 \mathrm{~ms}$ to $500 \mathrm{~ms}$ to remove boundary effects. For each experimental condition, frequency, and time instant separately, the phases $\varphi_{i k}$ at an electrode $i$ in a trial $k$ were taken as input to the procedures of phase synchronization analysis described above. That is to say, the electrodes were assumed to represent autonomous oscillators and the trials were treated as realizations of the considered stochastic process.

\section{Results}

To get an overview of the processes in the different frequency bands, we calculate three measures of the overall synchronization strength for each frequency and time instant: 1) The mean of the bivariate synchronization indices for all pairs of electrodes,

$$
\frac{2}{N(N-1)} \sum_{i, j>i} \bar{R}_{i j}
$$

2) the ratio of $\bar{R}_{i j}$ whose $t$-statistic difference from the value at $-150 \mathrm{~ms}$ exceeds a certain threshold, corresponding to a significance test at a level of 5\% (cf. Allefeld \& Kurths [this issue]), and 3) an estimate of the cluster strength

$$
r_{\text {Cluster }}=\frac{1}{\sum \mathrm{A}^{-1}\left(\rho_{j \mathrm{C}}\right)} \sum_{j} \mathrm{~A}^{-1}\left(\rho_{j \mathrm{C}}\right) \rho_{j \mathrm{C}} .
$$

The results for the Kanizsa condition are displayed in time-frequency plots in Fig. 2. All of the quantities reveal two distinct increases in synchronization related to the stimulus presentation, one below $10 \mathrm{~Hz}$ with a latency of 100-200 ms, and a second one around $13 \mathrm{~Hz}$ and a latency of about $300 \mathrm{~ms}$. Interestingly, there seem to be no other synchronization effects in the higher frequency bands. The increased values of mean and $r_{\text {Cluster }}$ around $50 \mathrm{~Hz}$ are a result of a direct influence of the power line onto the EEG voltage recordings; this effect disappears in the threshold ratio which describes the difference to the prestimulus level.

For a more detailed examination of the analysis results, we choose the band around $13 \mathrm{~Hz}$ of the higher-frequency response. Since the cluster analysis delivers indices $\rho_{i C}$ attributed to the electrodes, their values can be displayed in a conventional scalp map to give an easily apprehensible representation of the topographic information obtained. Figure 3 shows the time evolution of the synchronization topography for the Kanizsa condition. The emergence of the synchronization cluster around $150 \mathrm{~ms}$ can be clearly seen. Its distribution seems to be almost constant until its disappearance at $450 \mathrm{~ms}$ and involves mainly parietal as well as right fronto-temporal areas, with a maximum in the left parietal region.

Figure 4 shows a comparison of the three overall measures introduced above for the different experimental conditions. All of them indicate that there is a difference between the Kanizsa condition and the two other conditions. (A first statistical analysis of the threshold ratio at 300 ms shows strong significance $p=0.007$ for the difference between Kanizsa and Triangle and standard significance $p=0.05$ for the difference between Kanizsa and Non-Triangle conditions; for the other two measures the statistical differences are weaker.) Disregarding scaling, the information given by mean and $r_{\text {Cluster }}$ seems to be almost the same. This suggests that the generic analysis giving the $\rho_{i C}$ should possibly not be complemented by the index of cluster strength, whose estimation introduces further details of the dynamical model, but by the seemingly equivalent mean. The threshold ratio gives a slightly different time structure and has the advantage that there is no apparent difference 

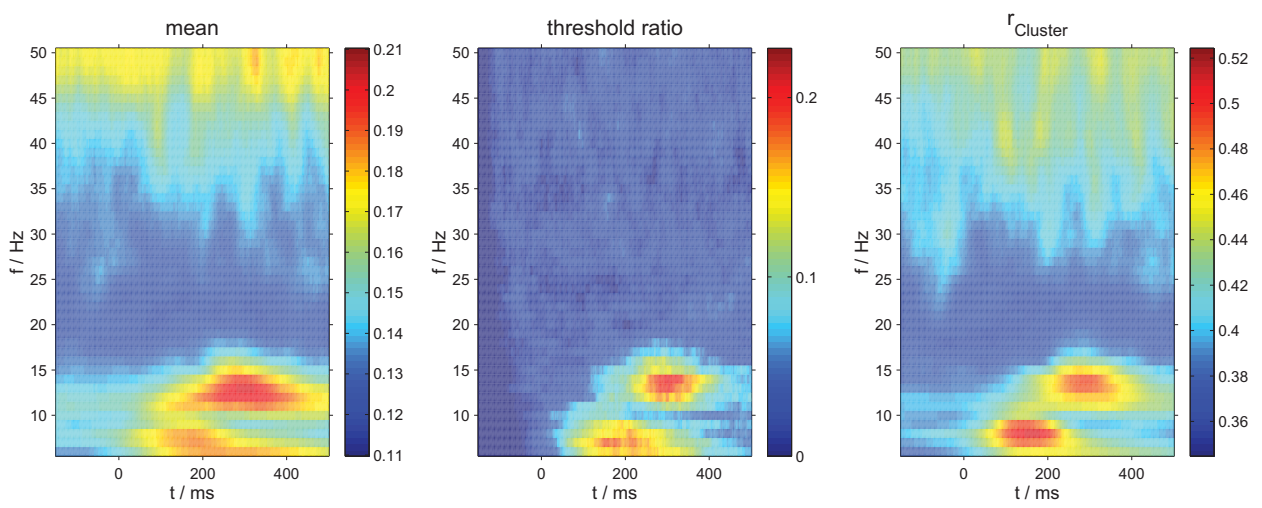

Figure 2: Time-frequency plots of three measures of the overall synchronization state for the Kanizsa condition.
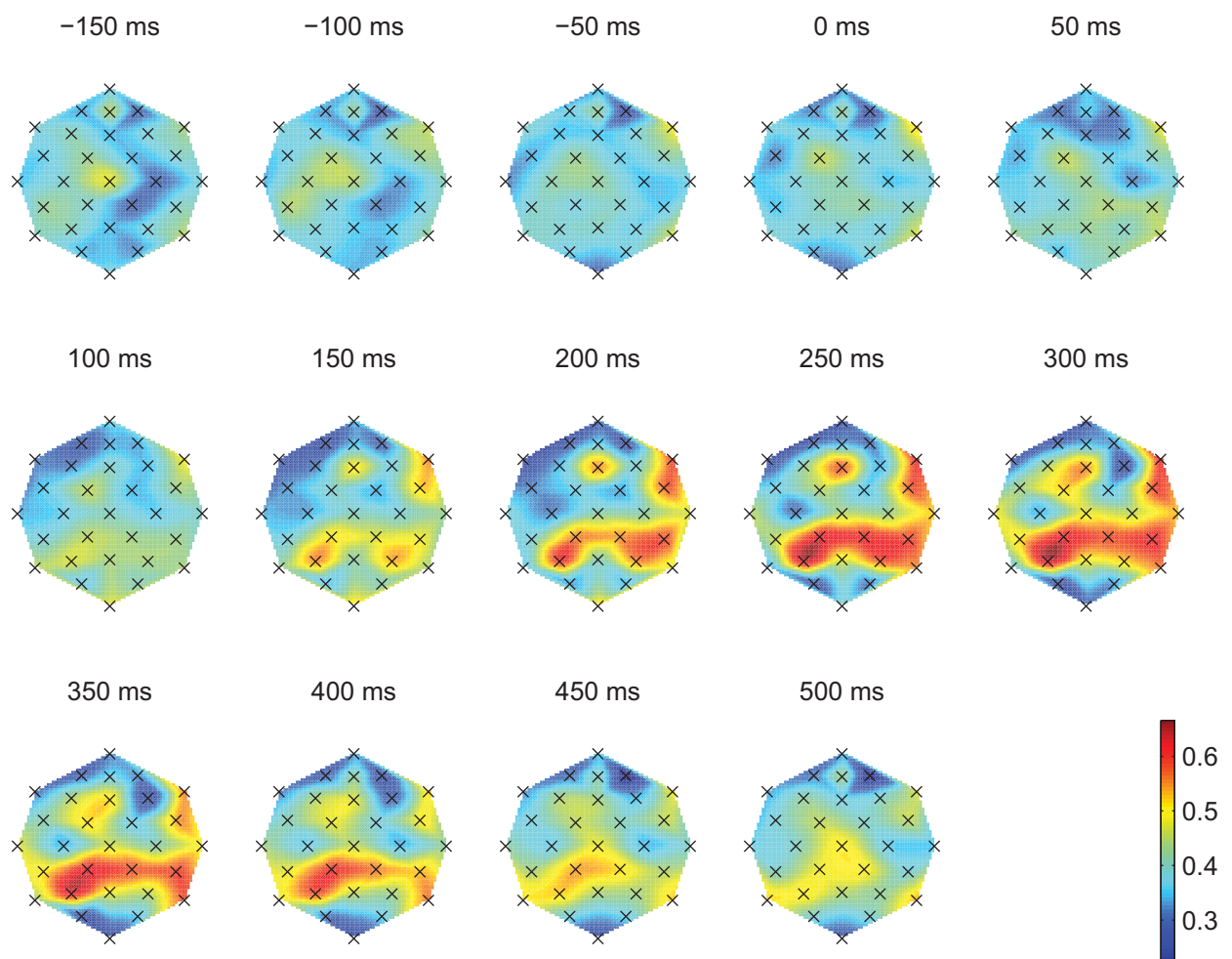

Figure 3: Time evolution of the cluster synchronization topography at $f=13 \mathrm{~Hz}$ for the Kanizsa condition. The continuous colors correspond to an interpolation of the $\rho_{i \mathrm{C}}$-values attributed to the electrodes, whose positions are marked by $\times$ symbols. For a chart of the electrode names, see Fig. 5. 
between conditions in the prestimulus interval (and even until $200 \mathrm{~ms}$ ). The different synchronization clusters in the three conditions at $300 \mathrm{~ms}$ are shown in Fig. 5. The basic topography seems to be the same in all conditions, with overall higher values in the Kanizsa condition. The residual errors $E_{i j}$ for these analyses are between -0.32 and 0.58 s.d.; these small values show that there is a basic agreement between model and data.

\section{Conclusion}

We have introduced an approach to the statistical phase synchronization analysis of multivariate data based on the concept of a synchronization cluster. The derived algorithm has been shown to use only a small number of assumptions about the dynamics underlying the data, which justifies its status as a generic data analysis. It has been applied to human EEG data from a visual attention psychological experiment, resulting in a common topography of synchronized behavior, but showing differences in the overall strength of synchronization between experimental conditions. The presented method is expected to give significant results in further EEG studies in the field of cognitive sciences, obtaining additional information on brain dynamics in a topographically, temporally, and frequency-specific way, as well as in other fields concerned with multivariate oscillatory processes.

The application of the method presented in this paper served to demonstrate its useability in the processing of empirical data; therefore we did not interpret our results with respect to physiological or cognitive processes or the results of other experiments following the same paradigm. Further studies trying to utilize our method in the examination of brain activation associated with cognitive processes are under way and will compare the results to other methods of EEG analysis. Complementing this, further work will investigate the analysis of data sets with multiple (disjunct or interacting) clusters and the statistical properties of the used measures of overall synchronization.

\section{Acknowledgments}

This work has been supported by Deutsche Forschungsgemeinschaft, research group "Conflicting Rules".

The author thanks the members of the group, part B1, for their help and Ina Bornkessel (MPI Leipzig) for her support in performing the experiment.

A MATLAB implementation of the SCA algorithm can be obtained from the corresponding author.

\section{References}

Allefeld, C. \& Kurths, J. [this issue] "Testing for phase synchronization" Int. J. Bifurcat. Chaos.

Haig, A. R., Gordon, E., Wright, J. J., Meares, R. A., \& Bahramali, H. [2000] “Synchronous cortical gamma-band activity in task-relevant cognition" Comput. Neurosci. 11(4), 669-675.

Kiss, I. Z. \& Hudson, J. L. [2001] "Phase synchronization and suppression of chaos through intermittency in forcing of an electrochemical oscillator" Phys. Rev. E 64, 6215. 

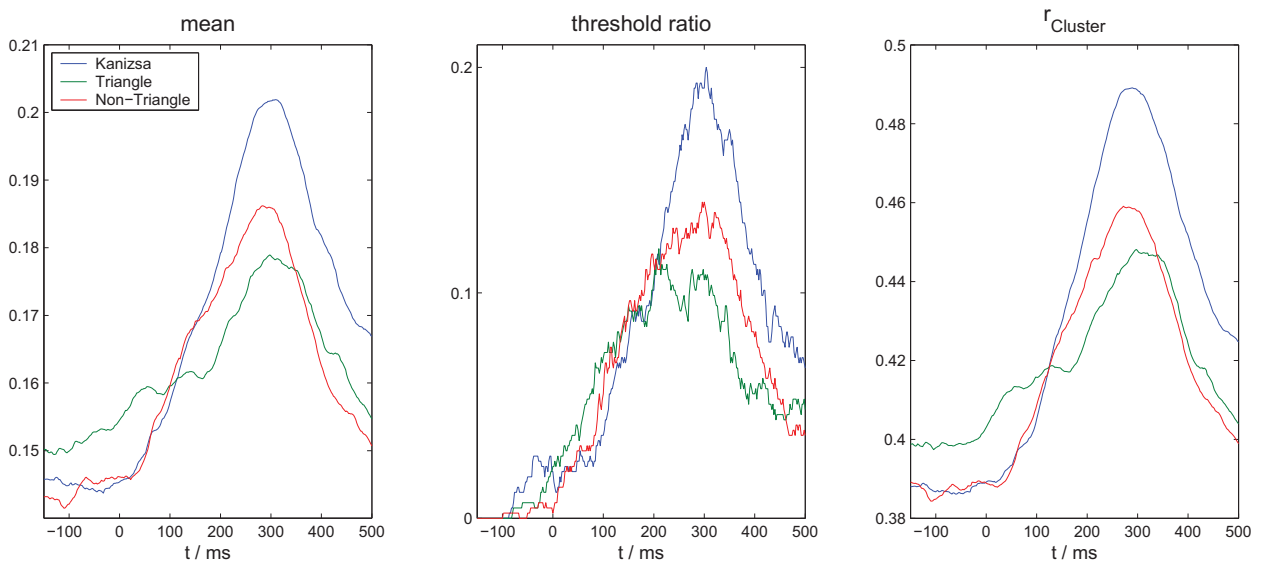

Figure 4: Differences in the time dependence of the three measures of overall synchronization at $f=13 \mathrm{~Hz}$ for the three experimental conditions: Kanizsa (blue), Triangle (green), and Non-Triangle (red).
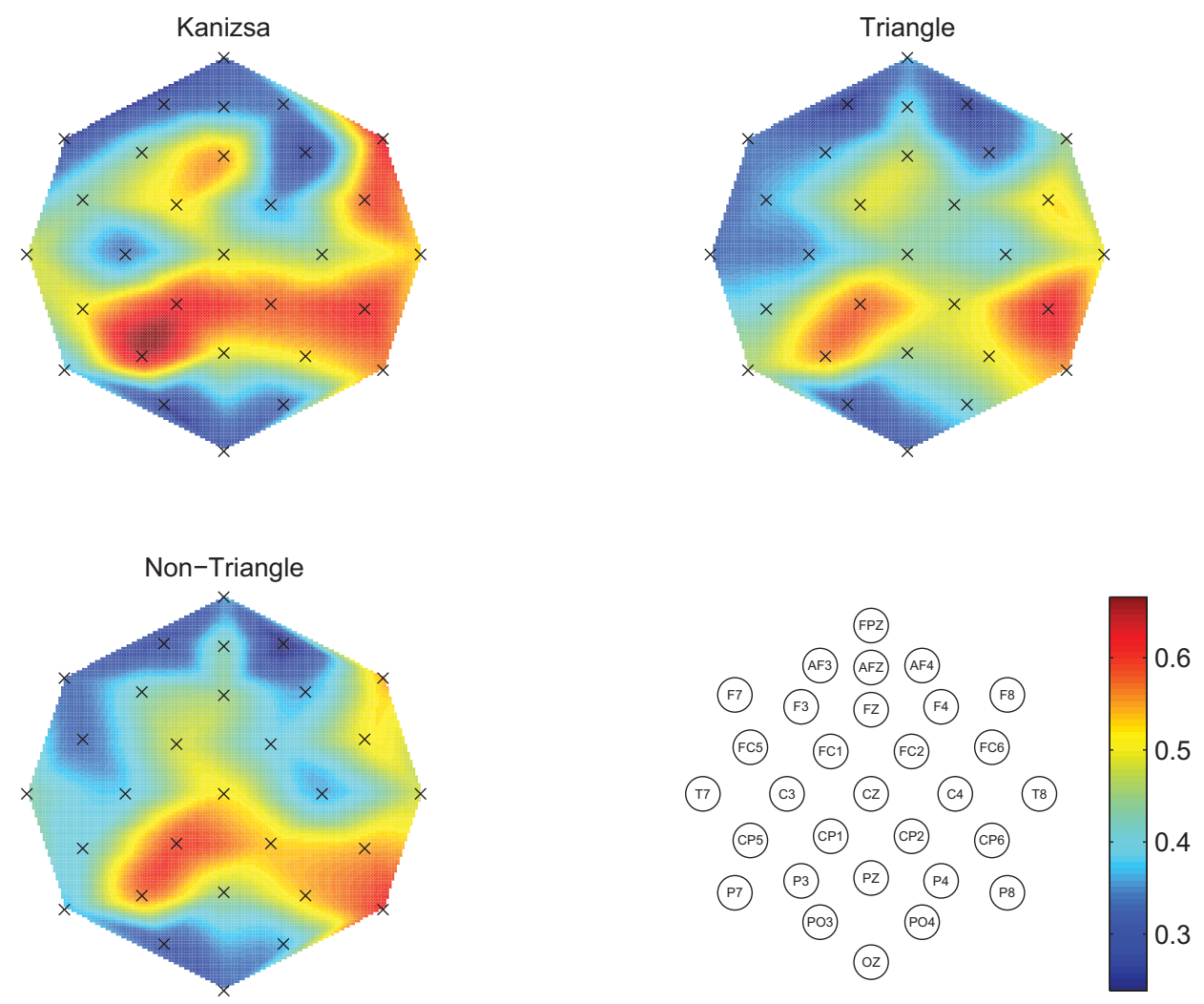

Figure 5: Differences in the synchronization topography at $f=13 \mathrm{~Hz}$ and $t=$ $300 \mathrm{~ms}$ for the three experimental conditions. 
Lachaux, J.-P., Rodriguez, E., Martinerie, J., \& Varela, F. J. [1999] “Measuring phase synchrony in brain signals" Hum. Brain Mapp. 8, 194-208.

Mardia, K. V. [1972] Statistics of Directional Data. (Academic Press).

Mardia, K. V. \& Jupp, P. E. [2000] Directional Statistics. (Wiley).

Mormann, F., Lehnertz, K., David, P., \& Elger, C. E. [2000] “Mean phase coherence as a measure for phase synchronization and its application to EEG of epilepsy patients" Physica D 144, 358-369.

Osipov, G. V. \& Kurths, J. [2001] “Regular and chaotic phase synchronization of coupled circle maps" Phys. Rev. E 65, 016216.

Osipov, G. V., Pikovsky, A. S., Rosenblum, M. G., \& Kurths, J. [1997] “Phase synchronization effects in a lattice of nonidentical Rössler oscillators" Phys. Rev. E 55(3), 2353-2361.

Parlitz, U., Junge, L., Lauterborn, W., \& Kocarev, L. [1996] “Experimental observation of phase synchronization" Phys. Rev. E 54(2), 2115-2117.

Perrin, F., Pernier, J., Bertrand, O., \& Echallier, J. F. [1989] "Spherical splines for scalp potential and current density mapping" Electroen. clin. Neuro. 72, 184-187 see also Corrigenda. ibid., 76, 565-566, 1990.

Pikovsky, A. S., Rosenblum, M. G., \& Kurths, J. [2001] Synchronization. A Universal Concept in Nonlinear Sciences. (Cambridge University Press).

Rodriguez, E., George, N., Lachaux, J.-P., Martinerie, J., Renault, B., \& Varela, F. J. [1999] "Perception's shadow: Long-distance synchronization of human brain activity" Nature 397, 430-433.

Rosenblum, M. G., Pikovsky, A. S., \& Kurths, J. [1996] "Phase synchronization of chaotic oscillators" Phys. Rev. Lett. 76, 1804-1807.

Strogatz, S. H. [2000] "From Kuramoto to Crawford: Exploring the onset of synchronization in populations of coupled oscillators" Physica D 143, 1-20.

Tallon-Baudry, C., Bertrand, O., Delpuech, C., \& Pernier, J. [1996] "Stimulus specificity of phase-locked and non-phase-locked $40 \mathrm{~Hz}$ visual responses in human" J. Neurosci. 16(13), 4240-4249.

Tass, P., Rosenblum, M. G., Weule, J., Kurths, J., Pikovsky, A. S., Volkmann, J., Schnitzler, A., \& Freund, H.-J. [1998] "Detection of $n: m$ phase locking from noisy data: Application to magnetoencephalography" Phys. Rev. Lett. 81(15), 3291-3294.

Ticos, C. M., Rosa, E., Pardo, W. B., Walkenstein, J. A., \& Monti, M. [2000] “Experimental real-time phase synchronization of a paced chaotic plasma discharge" Phys. Rev. Lett. 85, 2929-2932. 